\title{
Origin of resistivity in reconnection
}

\author{
Rudolf A. Treumann \\ Centre for Interdisciplinary Plasma Science, Max-Planck-Institute for extraterrestrial Physics, P.O.Box 1312, D-85741 Garching, Germany, and \\ International Space Science Institute, Hallerstr. 6, CH-3012 Bern, Switzerland
}

(Received June 5, 2000; Revised October 10, 2000; Accepted October 16, 2000)

\begin{abstract}
Resistivity is believed to play an important role in reconnection leading to the distinction between resistive and collisionless reconnection. The former is treated in the Sweet-Parker model of long current sheets, and the Petschek model of a small resistive region. Both models in spite of their different dynamics attribute to the violation of the frozen-in condition in their diffusion regions due to the action of resistivity. In collisionless reconnection there is little consensus about the processes breaking the frozen-in condition. The question is whether anomalous processes generate sufficient resistivity or whether other processes free the particles from slavery by the magnetic field. In the present paper we review processes that may cause anomalous resistivity in collisionless current sheets. Our general conclusion is that in space plasma boundaries accessible to in situ spacecraft, wave levels have always been found to be high enough to explain the existence of large enough local diffusivity for igniting local reconnection. However, other processes might take place as well. Non-resistive reconnection can be caused by inertia or diamagnetism.
\end{abstract}

\section{Introduction}

The central problem in the theory of reconnection concerns the violation of the frozen-in condition. This condition states that for large magnetic Reynolds numbers $R_{m}=\mu_{0} L V \sigma$ (or likewise large Lundkvist numbers which are the Alfvénic Reynolds numbers $R_{A}=\mu_{0} L \sigma V_{A}$ ) the plasma is effectively tied to the magnetic field such that the magnetic flux respectively the magnetic field lines move together with the plasma. For reconnection to work it is clear that this condition must be broken somewhere in a certain spatial region, the so-called diffusion region, in order to allow for the magnetic flux to be exchanged between two different types of plasmas. In other words, the magnetic field lines should be able to slip through the plasma and to rearrange into an X-point or X-line configuration as the one anticipated in the two basic reconnection models. Observations both in the laboratory as in space have accumulated sufficient convincing macroscopic evidence for reconnection to happen in various cases whenever the encountering plasmas contain antiparallel magnetic field components. Surprisingly little direct observational information has however been available about the structure of the diffusion region. In particular, in space plasma physics there is only indirect evidence available for reconnection. Either the actual diffusion region is very narrow even when it is extremely extended as required in Sweet-Parker's model of reconnection, or its signature in the particle, field and moment measurements has not been properly recognized. The latter seems highly probable and implies that it is still not known how the diffusion region should manifest itself in the observations. So far theory has not been able to provide a convincing picture of the structure of the diffusion

Copy right $(\mathrm{C}$ The Society of Geomagnetism and Earth, Planetary and Space Sciences (SGEPSS); The Seismological Society of Japan; The Volcanological Society of Japan; The Geodetic Society of Japan; The Japanese Society for Planetary Sciences. region even though a large number of extensive numerical simulations of reconnection have been performed including different spatial scales. Violation of the frozen-in condition classically implies the presence of one or the other diffusive process. It is thus of considerable interest to infer about the various possibilities of how sufficiently large diffusion can be generated in a plasma.

According to the type of diffusion one may distinguish resistive and non-resistive reconnection. Originally, SweetParker as well as Petschek reconnection refers to resistive or collisional reconnection with finite resistivity $\eta=\sigma^{-1}$ or otherwise finite collision frequency $v_{c}$. When reconnection shall be effective it is required that the bulk thermal plasma particle collisional mean free path $\lambda_{f}=1 / n S_{c}$ should be less than the linear extension $L$ of the diffusion region. Here $n$ is the particle number density, $v_{t}$ the thermal velocity, and $S_{c}$ is the collisional cross section. Equating $\lambda_{f}$ to the electron inertial length $c / \omega_{p e}$ sets a severe lower limit on the cross-section $S_{c}>\omega_{p e} / n c$. For densities $n \approx 30 \mathrm{~cm}^{-3}$ as in the magnetosheath close to the Earth's magnetopause this implies that $S_{c}>10^{-6} \mathrm{~cm}^{2}$ which is roughly 9 orders of magnitude larger than any reasonable collisional crosssection. One thus needs violently anomalous processes at work in order to enhance the cross-section to such values. Otherwise one must search for processes different from collisional which may set the frozen-in condition out of work. The possible processes can, in a fluid picture, be inferred from an inspection of the generalized Ohm's law of a plasma. It should, however, be noted that that Ohm's law belongs to the fluid concept identifying the resistivity as a macroscopic parameter. In a purely kinetic treatment one may thus expect that the concept of resistivity or diffusivity is not required. Kinetic reconnection may therefore be quite different from any of the macroscopic fluid models of reconnection. 


\section{Generalized Ohm's Law}

The generalized Ohm's law of a plasma is nothing else but the electron momentum conservation equation written in terms of the bulk plasma flow velocity $\mathbf{v} \approx \mathbf{j} /$ en $+\mathbf{v}_{e}$. In its most general version it reads (e.g. Krall and Trivelpiece, 1973)

$$
\begin{aligned}
\mathbf{E} & +\mathbf{v} \times \mathbf{B}-\eta \mathbf{j}=\frac{1}{\epsilon_{0} \omega_{p e}^{2}}\left\{\frac{\partial \mathbf{j}}{\partial t}+\nabla \cdot(\mathbf{j v}+\mathbf{v j})\right\} \\
& +\frac{1}{e n}\left\{\mathbf{j} \times \mathbf{B}-\nabla \cdot \mathrm{P}_{e}^{\mathrm{cm}}+\mathbf{F}_{e}^{\mathrm{pmf}}\right\} .
\end{aligned}
$$

Here the first braced term on the right accounts for electron inertia. The first two terms in the second braces have the structure of a generalized Hall term $\mathbf{j}_{\text {tot }} \times \mathbf{B} /$ en whence $\mathbf{j}_{\text {tot }}=\mathbf{j}+\mathbf{j}_{d}+\mathbf{j}_{c}$. The tensor pressure gradient term is recognized as the sum of the diamagnetic $\left(\mathbf{j}_{d}=-\mathbf{B} \times \nabla_{\perp} p_{\perp} / B^{2}\right)$ and curvature $\left(\mathbf{j}_{c}=-\left(p_{\perp}-p_{\|}\right) \mathbf{B} \times \mathbf{N} / B^{2} R_{c}\right)$ currents, respectively, with $\mathbf{N}$ the external normal of the curved magnetic field and $R_{c}$ the curvature radius of the field. In addition, any non-diagonal pressure terms may contribute as well to violation of the frozen-in concept. They can be caused, for instance, by FLR effects which can only be accounted for in a more complete gyro-kinetic theory (e.g. Frieman and Chen, 1982; Brizard, 1989, 2000; Cheng, 1991; Chen, 1999; Johnson and Cheng, 1997; Cheng and Johnson, 1999). F $\mathbf{F}_{e}^{\mathrm{pmf}}$ in Eq. (1) is the two-fluid ponderomotive force which accounts for the presence of inhomogeneous large-amplitude $\omega>\omega_{c i}$ wave turbulence (e.g. Lee and Parks, 1988).

The relevance of the Hall current effect (the terms in the second braces) in collisionless reconnection has been shown by Drake et al. (1994) (see also Drake (1995)). Hall currents are responsible for excitation of whistler turbulence which results in diffusive pitch-angle scattering of electrons. Correspondingly, the diamagnetic and curvature Hall currents are responsible for so far not investigated mirror and Alfvén wave turbulence (Treumann and Sckopke, 1999) in reconnection.

Ponderomotive effects have so far been ignored in any theory. They arise from the contribution of the average (meso-scale and large-scale) spatial and temporal variations of any high-frequency fluctuations in the plasma, and are therefore proportional to the average power-spectral densities of such variations. For instance, a spatially varying electrostatic high-frequency field $\mathbf{E}$ of frequency $\omega>1 / \tau$ and wavenumber $k>1 / L$, where $\tau$ is the slow time scale of variation and $L$ the typical spatial scale of variation in $\mathbf{x}$, exerts an average radiation pressure $P_{E}(\omega, \mathbf{k}, \mathbf{x})=\frac{1}{4} \epsilon_{0}[\epsilon(\omega, \mathbf{k})-$ $1]\left\langle|\mathbf{E}(\omega, \mathbf{k}, \mathbf{x})|^{2}\right\rangle$ on the plasma background. The angular brackets indicate small-scale averaging with respect to space, and $\epsilon(\omega, \mathbf{k})$ is the linear plasma response function. The negative gradient $-\nabla_{\mathbf{X}} P_{E}$ with respect to $\mathbf{x}$ of this pressure is the ponderomotive force density of the high frequency wave field. In this case the ponderomotive force can be written as a gradient field of a potential $P_{E} \equiv \phi^{\mathrm{pmf}}$. It simply adds to the diamagnetic or curvature current. Much more complicated expressions can be derived for any spatially and temporally varying wave field, electrostatic or electromagnetic. Of particular interest in reconnection though not yet investigated is the ponderomotive force effect of localized electromagnetic wave packets on the scale of the ion-inertial length, i.e. whistlers and kinetic (or shear) Alfvén waves. For such electromagnetic waves the force is not derivable from a ponderomotive potential.

All terms on the right of Eq. (1) are non-collisional. Violation of the frozen-in concept can nevertheless be caused in some specific ways by one or the other. The classical Hall term generates whistlers which pitch-angle scatter the electrons and sometimes cause remarkably fast particle diffusion across the field. The Alfvénic modes also scatter particles though to lesser extent. Their short wavelength branch merges with the whistler branch and extends the spectrum of the latter to low frequencies and long waves. The condition for their excitation is given by the firehose criterion (see also Hollweg and Völk, 1970). Ion or electron mirror modes are excited when the firehose criterion is set out of action and the plasma pressure is anisotropic with $p_{\perp}>p_{\|}$and $\beta$ sufficiently large. They are of interest because the frozen-in concept is violated in them by diamagnetic depletion of the magnetic field. However, none of these terms may generate anomalous collisions. When speaking of resistivity one is thrown back on the question of which processes contribute to the resistive term $-\eta \mathbf{j}$ on the left of Eq. (1).

\section{Anomalous Resistivity}

In space plasmas classical collisional times are extraordinarily long. Under the conditions of the near-Earth solar wind the collisional mean free path of a particle is about 5 AU and increases with heliocentric distance. In the tail plasmasheet it is even 10-20 times longer which entirely rules out Coulomb collisions to be of any importance on reconnection scales $<1 \mathrm{R}_{\mathrm{E}}$. Collisions must therefore necessarily be anomalous and mediated by the presence of high-frequency wave fields. The idea is that electrons interact with the selfexcited high-frequency wave spectrum until their orbits become stochastic. When averaging over the fluid time scale $\tau_{\text {fluid }} \gg \omega_{p e}, \omega_{c e}$ this interaction can be interpreted as friction which causes a difference in the bulk motion of electrons and ions or current $\left|\mathbf{v}_{i}-\mathbf{v}_{e}\right|=|\mathbf{j}| /$ en $=\eta_{\mathrm{a}}^{-1}|\mathbf{E}|$, with anomalous resistivity defined by the classical formula

$$
\eta_{a}=v_{a} / \epsilon_{0} \omega_{p e}^{2} .
$$

Here $v_{a}$ is the anomalous collision frequency. It depends on the very mechanism of the wave particle interaction and is a functional of the self-excited wave energy spectrum $W_{k}$. To obtain an idea of the relation between the collision frequency and the wave spectrum one may realize that the classical Spitzer-Braginskii collision frequency can be written (e.g. Treumann and Baumjohann, 1997, p. 321) as

$$
v_{c}=\omega_{p e} W_{\mathrm{th}} \ll \omega_{p e}, \quad W_{\mathrm{th}} \simeq\left(n \lambda_{D}^{3}\right)^{-1} .
$$

It turns out to be directly proportional to the thermal fluctuation level $W_{\text {th }} \ll 1$ in non-magnetized plasma waves. This level is in general very low and thus inefficient in scattering the electrons in collisionless plasmas. However, one immediately recognizes that whenever the fluctuation level is increased in the presence of instability, $v_{a}$ can occasionally exceed $v_{c}$ by many orders of magnitude. A very general expression for the anomalous collision frequency is thus

$$
v_{a}=\omega_{p e} W_{\text {turb }}
$$




$$
W_{\mathrm{turb}}=\frac{\epsilon_{0}\left\langle\epsilon\left(\omega_{k}, \mathbf{k}\right)\left|\mathbf{E}_{\mathbf{k}}\right|^{2}\right\rangle}{2 n k_{B}\left(T_{e}+T_{i}\right)} \gg W_{\mathrm{th}}
$$

where $\mathbf{E}_{k}$ is the turbulent plasma wave electric field, $\epsilon\left(\omega_{k}, \mathbf{k}\right)$ is the nonlinear plasma response function, and the angular brackets indicate averaging over the whole spectrum of fluctuations. Usually the response function is calculated from linear theory. This is in contrast to the Spitzer-Braginskii expression which was based on the exact fluctuation level in thermal equilibrium. Hence, in a theory of comparable precision the fully nonlinear (renormalized) response function should enter the above expression as well as the average final stationary turbulent fluctuation spectrum should be inserted for $W_{\text {turb }}$. In general such a calculation is impossible for many reasons. The most obvious one is that the fastest linearly growing mode which is usually taken as the most active one readily achieves nonlinear amplitudes where it either saturates, scatters or traps the electrons. At this stage slower growing modes may become important in addition to nonlinear sidebands which can be pumped by the originally growing wave. All these interactions render the exact problem untractable. In some very simple cases it is possible to extend the fluctuation theory to include more complicated stable states. Figure 1 shows the thermal fluctuation level of an unmagnetized one-dimensional electron beam-plasma interaction for different beam-to-plasma temperature ratios $T_{b} / T_{e}$. The basic mode in this system is the Langmuir mode which in presence of the beam attains an increase in fluctuation energy which depends on the beam temperature $T_{b}$. For long wavelengths $\lambda>\lambda_{D}$ the increase in the fluctuation level is about proportional to the temperature ratio. This implies that an enormous beam temperature would be required to yield the wanted classical Spitzer-Braginskii collision frequency. Since this will not be practical, a nonlinear theory is needed. Free energy for an instability can be provided by currents $\mathbf{j}$ (relative drifts between electrons and ions: $\mathbf{v}_{D}$ in the ion frame) parallel or perpendicular to the magnetic field or the various gradients $\nabla n, \nabla T, \nabla \mathbf{v}, \nabla_{\mathbf{x}, \mathbf{v}} f_{e}(\mathbf{x}, \mathbf{v})$ of density, temperature, by velocity shear, or by spatial or phase space gradients of the electron distribution function $f_{e}(\mathbf{x}, \mathbf{v})$. This implies a large number of linear instabilities not all of which may be of relevance in the problem of anomalous resistivity.

In weak turbulence the unstable modes evolve according to the nonlinear wave equation

$$
\frac{\partial W_{k}}{\partial t}=2 \gamma_{k}\left(\omega_{k}, t\right) W_{k}+A_{k} W_{k}^{2}+\cdots
$$

where the number of relevant terms in the expansion on the right depends on the dispersion of the wave. The coefficient $A_{k}$ (and all following coefficients) are complicated functions describing the mutual interaction of wave modes of different wave numbers $k, k^{\prime}, \ldots$ and/or the interaction between waves and particles. Now, assuming $W_{k}$ and the nonlinear growth rate are known then, in equilibrium, the anomalous collisional change $m_{e} n v_{a} \mathbf{v}_{D}=-e n \mathbf{E}$ in plasma momentum should balance the change in wave momentum

$$
\Delta \mathbf{p}_{\text {turb }}=\int \frac{\mathrm{d}^{3} k}{8 \pi^{3}} \frac{\mathbf{k}}{\omega_{k}} \gamma(\mathbf{k}) W_{k} .
$$
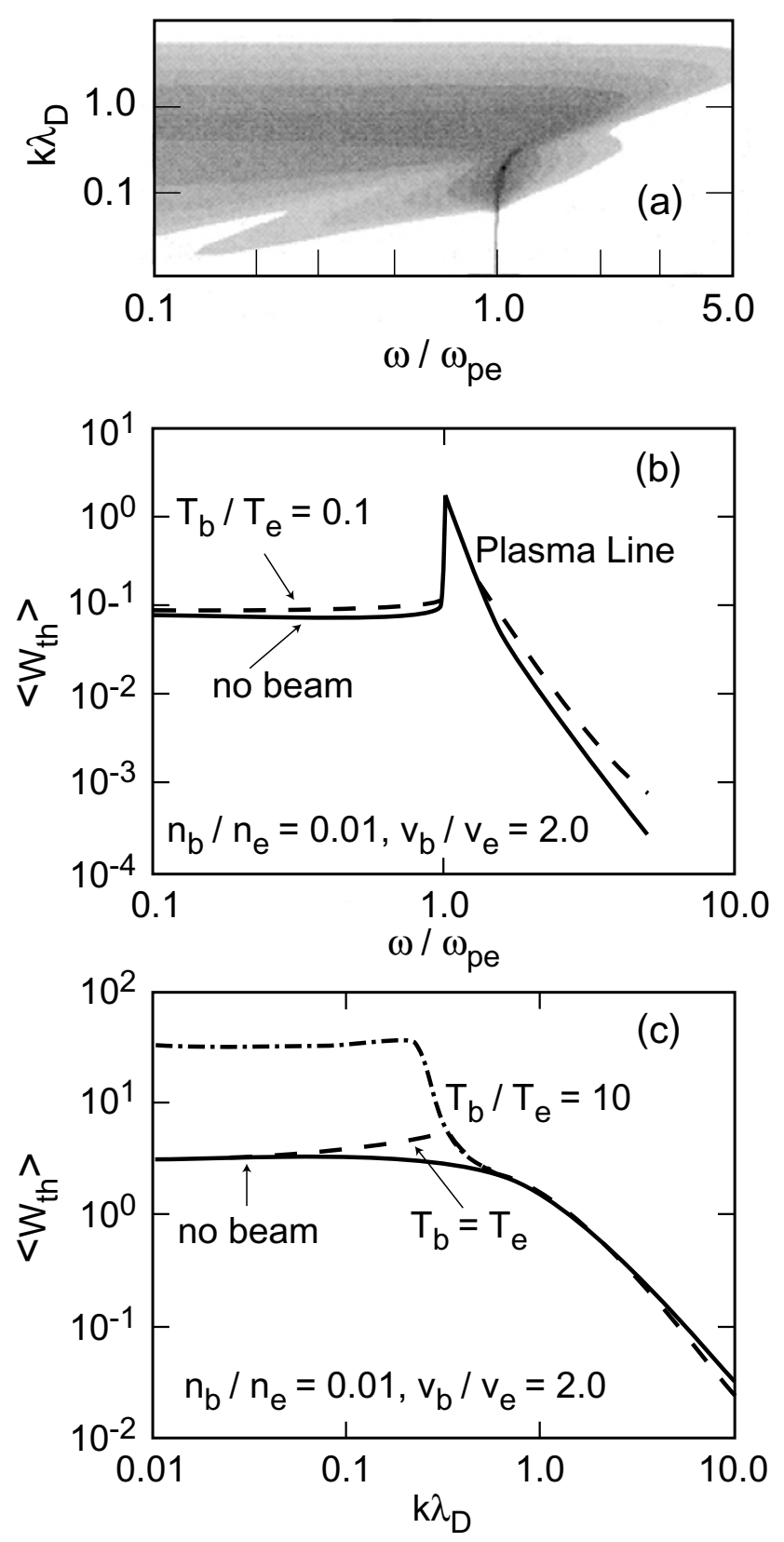

Fig. 1. Plasma wave thermal fluctuation level in a beam-plasma system. (a): dispersion of Langmuir and ion-acoustic beam fluctuations for the beam to plasma density ratio is $n_{b} / n_{e}=0.001$. (b): Integrated over $k$ intensity as function of frequency and (c): integrated wave power as function of $k$ for $n_{b} / n_{e}=0.01$ and the beam speed is $v_{b}=2.0 v_{e}$. At large beam temperatures, the fluctuation level of long-wavelength plasma fluctuations increases about proportional to the beam to plasma temperature ratio $T_{b} / T_{e}$ (after Lund et al., 1996).

It then follows immediately that

$$
v_{a}=\frac{1}{n m_{e} v_{D}^{2}} \int \frac{\mathrm{d}^{3} k}{8 \pi^{3}} \frac{\mathbf{k} \cdot \mathbf{v}_{D}}{\omega_{k}} \gamma(\mathbf{k}) W_{k}
$$

is the general formal weak turbulence expression for the anomalous collision frequency. Note, however, that this expression implies knowledge of the weakly turbulent wave spectrum, the near equilibrium growth rate and also the reaction of the wave spectrum on the current drift velocity which itself is a functional of the collisional interaction. In this gen- 
erality the above formula is nothing but a formal vehicle. It becomes useful only in the quasi-linear approximation where the growth rate is taken in linear approximation, and the drift velocity is assumed constant. In addition, the above expression ignores any strongly turbulent effects like those which have been mentioned above. The above formula can, in an average sense, be rewritten into a form suitable for simple estimates:

$$
v_{a} \approx \frac{\epsilon_{0}|\mathbf{E}|^{2}}{2 n m_{e} v_{D}}\left[\frac{\partial \omega \epsilon(\omega)}{\partial \omega}\right]_{\max }\left[\frac{k(\omega) \gamma(\omega)}{\omega}\right]_{\max }
$$

where the index max indicates that all quantities have to be taken at the maximum unstable wave number $k_{\max }(\omega)$.

The conditions determining the relevance of a certain wave mode at the reconnection diffusion site, are as follows: (1) the plasma is weakly magnetized only, $\left(\omega_{p e} \gg \omega_{c e}\right)$; (2) in the absence of a strong guide magnetic field, one has $\beta \gg 1$, implying that the plasma is hot and must hence be treated by kinetic theory; (3) the drift velocity (current) is susceptible or even high (strong currents); (4) spatial gradients of the plasma parameters $n, T_{e}, T_{i}, p_{e}$ and of the distribution functions cannot be neglected a priori on scales smaller than and of the order of the ion (electron) inertial length $\lambda_{i}=c / \omega_{p i}\left(\lambda_{e}=c / \omega_{p e}\right) ;(5)$ in application to reconnection in the Earth's tail plasma sheet one encounters the additional restriction that the electron temperature is lower than the ion temperature, $T_{i} \simeq 7 T_{e}$, and $k_{B} T_{i} \approx 1 \mathrm{keV}$. Reviews of average plasma wave spectra in the magnetospheric tail plasma sheet, identifying some of the modes which may be of relevance in reconnection, have been given by Baumjohann et al. (1989, 1990).

\subsection{Buneman mode}

The above condition (5) imposes a severe restriction on the wave mode responsible for generation of resistivity. At temperatures $T_{e} \leq T_{i}$ the ion-acoustic wave mode is theoretically stable. This is unfortunate because in the presumably nearly unmagnetized plasma of the inner diffusion region one expects ion-acoustic waves to be generated first. Since this is ruled out, the most important instability will become the Buneman mode. This is a reactive instability that sets on at very large current drifts $v_{D}>v_{e}$. The above ion-to-electron temperature ratio still implies $v_{e} \approx 16 v_{i}$. It is not known whether the currents can become strong enough to exceed this threshold in reconnection. Whenever it may happen, the excited wave is negative energy and of phase speed $\left|\omega_{B} / k\right| \simeq v_{D}\left(m_{e} / 2 m_{i}\right)^{1 / 3} \approx 0.06 v_{D}$. Since $k \simeq \omega_{p e} / v_{D}$, and $\gamma_{B} \simeq \sqrt{3} \omega_{B}$, the Buneman frequency is $\omega_{B} \simeq \omega_{p e}\left(m_{e} / 16 m_{i}\right)^{1 / 3} \approx 0.03 \omega_{p e} \approx \gamma_{B}$. The instability is fast growing and high frequency, $\omega_{p i}<\omega_{B}<\omega_{p e}$. The linear growth time is $\tau_{B} \simeq 20 / \omega_{p e}$. On this short time scale the amplitude of the wave assumes large values, and the wave starts trapping the current electrons and slows them down to the wave phase velocity, less than threshold. The Buneman instability quenches itself by disrupting the current in a time $\tau \simeq\left(2 m_{i} / 3 m_{e}\right)^{1 / 3} / \omega_{p e} \approx 10 / \omega_{p e}$. The estimated plasma sheet wave energy density becomes $\left\langle W^{B}\right\rangle \leq T_{i} / T_{e} \approx 7$. This leads to a Buneman collision frequency of

$$
v_{a}^{B} \simeq \omega_{p e} \sqrt{m_{e} / m_{i}}=\omega_{p i}
$$

which yields a value $10^{7}$ times the classical collision frequency, roughly what is needed. The energy of the current is imposed into electron heating which proceeds in a time $\tau_{\text {heat }}^{B} \sim \omega_{p i}^{-1}=43 / \omega_{p e}$. The effect of the Buneman instability is to prepare the plasma to become ion-acoustic wave unstable when the plasma is heated temporarily to electron temperatures $T_{e}>T_{i}$. One may thus argue that the diffusion region when becoming Buneman-unstable increases the electron temperature locally in a bursty manner above the ion temperature subsequently driving ion-acoustic waves unstable. There is no experimental verification of this effect in the reconnection region.

An additional effect implicit to the Buneman instability is electron trapping. This trapping affects almost all the low energy electrons. When heated, the faster ones escape the wave. One therefore expects that a Buneman instability will in saturation lead to formation of BGK modes. These may act as a source of energetic run-away electrons in reconnection.

\subsection{Ion-acoustic turbulence}

Whence the electron temperature is high enough, the ionacoustic instability takes over. Historically the first step into the direction of estimating the anomalous resistivity in an unstable plasma has been made by Sagdeev (1966) on taking advantage of this basic non-magnetized plasma mode. Like the Buneman instability, it is driven by the relative drift $\mathbf{v}_{D}$ between electrons and ions but for lower drift speeds $c_{s}<$ $v_{D}<v_{e}$. Its dispersion relation is given by

$$
\omega_{k}^{s}=k c_{s} / \sqrt{1+k^{2} \lambda_{D}^{2}}, \quad c_{s}^{2}=2 k_{B}\left(T_{e}+T_{i}\right) / m_{i} .
$$

This dispersion relation does not allow for three wave interaction. Hence, to lowest approximation the wave-wave interaction is determined by the very weak (because of $B_{k}$ being small) third order term in

$$
\partial_{t} W_{k}^{s}=2 \gamma_{k}^{s} W_{k}^{s}-A_{k} W_{k}^{s 2}+B_{k} W_{k}^{s 3} .
$$

Thus the most important nonlinear interaction at this stage that stabilizes the wave is induced scattering (the second order term) at thermal ions, resulting from $\omega_{1}-\omega_{2}=\mathbf{v}$. $\left(\mathbf{k}_{1}-\mathbf{k}_{2}\right)$. Neglecting $B_{k}$ and writing the growth rate $\gamma_{k} \approx$ $\left(v_{D} / v_{e}\right) \omega_{k}$, one finds the total wave power in equilibrium just to be $\left\langle W^{s}\right\rangle \approx\left(T_{e} / T_{i}\right)\left(v_{D} / v_{e}\right)$. The Sagdeev formula for the ion-acoustic anomalous collision frequency becomes

$$
v_{a}^{s} \simeq 0.01 \omega_{p i}\left(T_{e} / T_{i}\right)\left(v_{D} / c_{s}\right) \text { for } v_{D} / c_{s}<6 .
$$

The last condition results from avoiding Landau damping of the waves which generates a high energy tail of the form

$$
f_{i}(v) \propto \exp \left(-\alpha v^{5}\right)
$$

on the distribution function (e.g. Dum, 1978). The average energy of these tail ions is $\left\langle E_{i \mathrm{~T}}\right\rangle \sim k_{B} T_{e}$ comparable to the electron thermal energy. Finally, when the third-order term comes into play, the collision frequency reads

$$
v_{a}^{s} \simeq\left(\pi / k \lambda_{D}\right)\left(v_{e} / v_{D}\right)^{3}\left\langle W^{s}\right\rangle
$$

which decreases as the third power of the drift velocity. The anomalous collision frequency thus has a maximum $\nu_{a \max }^{s} \approx$ $0.1 \omega_{p e}$ at a drift speed of about $v_{D \max } \simeq\left(m_{i} / m_{e}\right)^{1 / 6} c_{s}=$ 
$3.5 c_{s}$. In the tail reconnection region, assuming an electron density of roughly $n \approx 1 \mathrm{~cm}^{-3}$ and an electron temperature of $k_{B} T_{e} \sim 1 \mathrm{keV}$, this yields a maximum theoretical anomalous ion-acoustic collision frequency of about $\nu_{a}^{s} \sim 5-6 \mathrm{kHz}$ for a current drift speed of $v_{D} \sim 1000 \mathrm{~km} \mathrm{~s}^{-1}$ or current density of $|\mathbf{j}| \sim 1.5 \times 10^{-7} \mathrm{~A} \mathrm{~m}^{-2}$ in the diffusion region. The corresponding diffusion region $\mathrm{Ohm}$ electric field follows from $E \sim \eta_{a}^{s}|\mathbf{j}| \approx 20 \mathrm{mV} \mathrm{m}^{-1}$. A collision frequency of this value should be sufficient to drive reconnection. It requires, however, the observation of strong ion acoustic turbulence of the order of energy level $\left\langle W^{s}\right\rangle \simeq 0.01$ in the reconnection site, where we assumed an ion-to-electron temperature ratio of $T_{i} / T_{e} \simeq 7$ as frequently observed. Such high wave levels have so far never been observed which puts some doubt in the validity of the above estimates and in the applicability of this theory.

Doubts in the validity of the linear/quasilinear theory do also arise when considering the large $T_{i} / T_{e}$ ratio. Linear theory suggests that the ion-acoustic instability is very hard to excite under such conditions unless the electron current drift $v_{D} \sim v_{e}$ approaches the electron thermal speed which happens only for very low electron temperatures. Since at the reconnection site electrons are believed to be heated, this is not expected to hold. The above mentioned interplay between Buneman and ion-acoustic modes could circumvent the problems by heating the electrons on a short time scale for current drift that high. However, as discussed before, there is little indication for conditions under which the Buneman instability would work here. Moreover, the observed ion-toelectron temperature ratio argues against such an interplay. One may thus tend to abandon the ion-acoustic mode.

It should, however, be noted that in simulations the evolution of ion-acoustic turbulence has been found to differ substantially from simple linear and quasi-linear instability theory. Ion-acoustic waves readily evolve into ion phasespace holes or ion BGK modes (Gray et al., 1991; Tetreault, 1991). Thereby the ion-acoustic instability threshold is considerably reduced, and saturation of the ion-acoustic wave is obtained at much lower average wave levels. Accordingly an even lower anomalous resistivity may be caused.

\subsection{Strong Langmuir turbulence}

Since thermal Langmuir fluctuations are responsible for classical collisions one may expect that strong Langmuir turbulence may have an important effect on enhanced anomalous resistivity. This is indeed the case. However, as we demonstrate, the estimated resistance is insufficient for reconnection. Accounting for the ponderomotive force, Langmuir turbulence at large amplitudes is described by the Zakharov equations (see Robinson, 1997)

$$
\begin{aligned}
& \partial_{t} \frac{\delta n}{n} \sim \frac{\epsilon_{0}}{4 m_{i}} \nabla^{2}\left\langle W^{L}\right\rangle, \quad\left\langle W^{L}\right\rangle \propto\left\langle\left|\mathbf{E}_{k}^{L}\right|^{2}\right\rangle \\
& \nabla \cdot\left[\partial_{t}+\frac{3}{2} \omega_{p e} \lambda_{D}^{2} \nabla \nabla \cdot\right] \mathbf{E}^{L}=\frac{\omega_{p e}}{2} \nabla \cdot\left(\mathbf{E}^{L} \frac{\delta n}{n}\right) .
\end{aligned}
$$

These equations include the mean reaction of the unstable fluctuations whose energy density cannot be neglected anymore on the plasma. Dimensionally they yield for the density variation and total average Langmuir wave power density, re- spectively

$$
\delta n \propto\left(\tau_{\mathrm{cav}}-t\right)^{-\frac{4}{d}}, \quad\left\langle W^{L}\right\rangle \propto \ell^{-d} \propto\left(\tau_{\mathrm{cav}}-t\right)^{-2}
$$

where $L$ designates Langmuir waves, $\ell \propto k^{-1}$ is the spatial length, $d$ spatial dimension and $\tau_{\text {cav }}$ is the collapse time when the waves form cavitons (or configuration space holes in the density) in which they are trapped. On using Kolmogoroff's assumption $W_{k}^{L} \mathrm{~d} k / \mathrm{d} t=$ const on the inertial range evolution of the wave spectral energy density one obtains the scaling of $W_{k}$ with $k$

$$
W_{k} \propto k^{-\left(1+\frac{1}{2} d\right)} .
$$

From modulational instability theory one finds that the caviton formation time $\tau_{\mathrm{cav}}=\gamma_{\mathrm{cav}}^{-1}$ can be found from

$$
\gamma_{\mathrm{cav}}=\omega^{p i} \sqrt{\left\langle W^{L}\right\rangle}
$$

Balancing the power $\gamma_{\text {cav }}\left\langle W^{L}\right\rangle$ absorbed by the particles by the collisional loss $v_{a}^{\text {cav }}\left[\epsilon_{0} / 2 n k_{B} T_{e}\right]\left\langle\left|\mathbf{E}^{L}\right|^{2}\right\rangle$, one may find an expression for the stationary wave power (e.g., Sagdeev, 1979 ) in continuously driven caviton formation and absorption,

$$
\left\langle W^{L}\right\rangle \sim \alpha \frac{m_{i}}{m_{e}}\left(\frac{\epsilon_{0}\left\langle\left|\mathbf{E}^{L}\right|^{2}\right\rangle}{2 n k_{B} T_{e}}\right)^{2}
$$

which when inserted into the balance expression yields the caviton-electron collision frequency

$$
\nu_{a}^{\mathrm{cav}} \sim \alpha^{\frac{3}{2}} \omega_{p i}\left(\frac{\epsilon_{0}\left\langle\left|\mathbf{E}^{L}\right|^{2}\right\rangle}{2 n k_{B} T_{e}}\right)^{2} .
$$

Here $\alpha=O(1)$ is a numerical factor which must be determined by numerical solution of the full Zakharov equations (e.g. Newman et al., 1994; Robinson, 1997). The collision frequency is proportional to the 4 th power of the wave field. In the final state of the collapse when the field power density becomes locally large, it may become substantial. But assuming that the relative power density stays at order $10^{-3}$, the collision frequency is of order $v_{a}^{\text {cav }} \simeq 10^{-6} \omega_{p i}$ which in the plasma sheet is only the order of $\sim 1 \mathrm{~Hz}$. Though this is a high collision rate in collisionless plasma it is lower than other collision rates that can be expected. In addition one may ask what the source of the Langmuir waves is. The most probable mechanism is beam generation of Langmuir waves. This requires acceleration of electron beams in reconnection which again requires other additional mechanisms to act at the same time. Thus observation of electron beams from the diffusion site in reconnection will assure one that Langmuir caviton formation takes place and that collision frequencies of the above estimated order of magnitude will be generated. Transit time acceleration of electrons in cavitons during their further evolution will then cause electron heating and acceleration into secondary beams.

\subsection{Lower-hybrid resistivity}

The reconnection diffusion region may be magnetized as the magnetic field may not be entirely, i.e. only partially annihilated even in the narrow diffusion region on either of scales $\lambda_{e}, \lambda_{i}$. In such a case reconnection takes place in presence of a guide field. As an alternative, closer to the boundaries of the reconnection region, the magnetic field is definitely non-zero. In the simplest model, its strength as function of 
the coordinate $z$ is given by the Harris current sheet model. The (X-line) current responsible for reconnection flows in the direction perpendicular to the magnetic field. Currents of this kind, when exceeding a relatively weak instability threshold, excite the so-called modified two-stream instability (e.g. Krall and Liewer, 1971) which has dispersion relation similar to that of the Buneman instability but this time including the ion inertia. In the case of a cold plasma with perpendicular current drift velocity $v_{D}$, the instability is nearly perpendicular to the magnetic field $\left(\tan ^{2} \theta \simeq m_{i} / m_{e}\right)$ with frequency and growth rate $\omega^{\mathrm{mts}} \simeq \gamma^{\mathrm{mts}} \approx \omega_{l h} / 2$, and $\omega_{l h}$ the lower-hybrid resonance frequency.

This modified two stream instability is a special case of a more general instability that arises in magnetized inhomogeneous plasmas. In the presence of pressure gradients the plasma self-consistently produces diamagnetic pressure and curvature drift currents which constitute the perpendicular current in the plasma. The resulting instability is known as the lower-hybrid drift instability (e.g. Liewer and Krall, 1973).

The lower-hybrid drift instability is a special case of a drift wave and is probably one of the most important instabilities in contributing to anomalous transport. It is excited in regions of comparably low $\beta$ where the magnetic field is still strong enough to render electrons magnetized while ions are already unmagnetized. It is hence the ideal instability at the boundaries of the diffusion region (or in presence of a weak magnetic guide field even in the center of the diffusion region).

The most interesting fact about this wave is that the plasma contributes to wave power density via the linear response function $\epsilon(\omega, \mathbf{k})$ such that

$$
\left\langle W^{\mathrm{lh}}\right\rangle \approx \frac{\epsilon_{0}}{2}\left(1+\frac{\omega_{p e}^{2}}{\omega_{c e}^{2}}\right) \frac{\left\langle\left|\mathbf{E}^{\mathrm{lh}}\right|^{2}\right\rangle}{n k_{B}\left(T_{e}+T_{i}\right)} .
$$

This is important when calculating the anomalous collision frequency. Maximum growth implies $\gamma^{\text {lh }} \simeq 0.3 \omega_{l h}\left(v_{D} / v_{i}\right)^{2}$ with $v_{i}$ the ion thermal velocity. Hence, the growth rate can become large when the drift speed exceeds the ion thermal speed. The collision frequency generated in lower-hybrid drift wave turbulence is

$$
v_{a}^{\mathrm{lh}} \simeq \omega_{l h} \sqrt{\frac{\pi}{8}} \frac{m_{i}}{m_{e}}\left\langle W^{\mathrm{lh}}\right\rangle .
$$

In this formula we have expressed the collision rate through the electric wave intensity which is a measurable quantity. The proportionality to the ion-to-electron mass ratio increases the collision frequency even for low average lowerhybrid wave energy density. Measurement of weak wave electric field intensities does therefore not necessarily imply that lower-hybrid waves are unimportant. Quasi-linear estimates have been given by Davidson (1978). These estimates of the saturation wave field, when compared with real measurements, have all turned out way too optimistic.

Indications of the presence of lower-hybrid turbulence in the reconnection region is sparse. The measurements of electrostatic waves in the magnetotail plasma sheet (Baumjohann et al., 1989, 1990) show an indication of enhanced intensites when approaching the lower hybrid frequency. Unfortunately, measuring low-frequency electric wave fields is difficult. One may expect better results from the upcoming CLUSTER missions. For slightly oblique propagation the lower-hybrid drift wave attains a weak magnetic component.

Figure 2 (Bauer et al., 1995) shows low-frequency magnetic power spectra taken in the central plasma sheet during magnetospheric substorm times when reconnection is expected to proceed in the near-Earth tail, compared to quiet time spectra. The substorm spectra above a frequency of $>1 \mathrm{mHz}$ exhibit strongly enhanced wave power (with power law decay to higher frequencies). The lower-hybrid frequency here is less than $\sim 0.2 \mathrm{~Hz}$ roughly coinciding with the observed increase in wave power. This is a strong argument for the presence of lower-hybrid turbulence in the interior plasma sheet. Note also that it had been found that this turbulence is weaker at the boundaries of the plasma sheet.

Low frequency magnetic turbulence has been detected by GEOTAIL in relation to reconnection in the distant tail (Hoshino et al., 1994) and has been confirmed by hybrid simulations (Arzner and Scholer, 2001). Transport near the $\mathrm{X}$-line has also been considered by Cai et al. (1994). Figure 3 shows the application of the lower-hybrid collision theory to the reconnection region at the magnetopause in dependence on the measured lower-hybrid wave electric field intensity. The perpendicular diffusion coefficient

$$
D_{\perp}^{\mathrm{lh}}=\frac{k_{B} T_{e}}{m_{e}} \frac{v_{a}^{\mathrm{lh}}}{\omega_{c e}^{2}}
$$

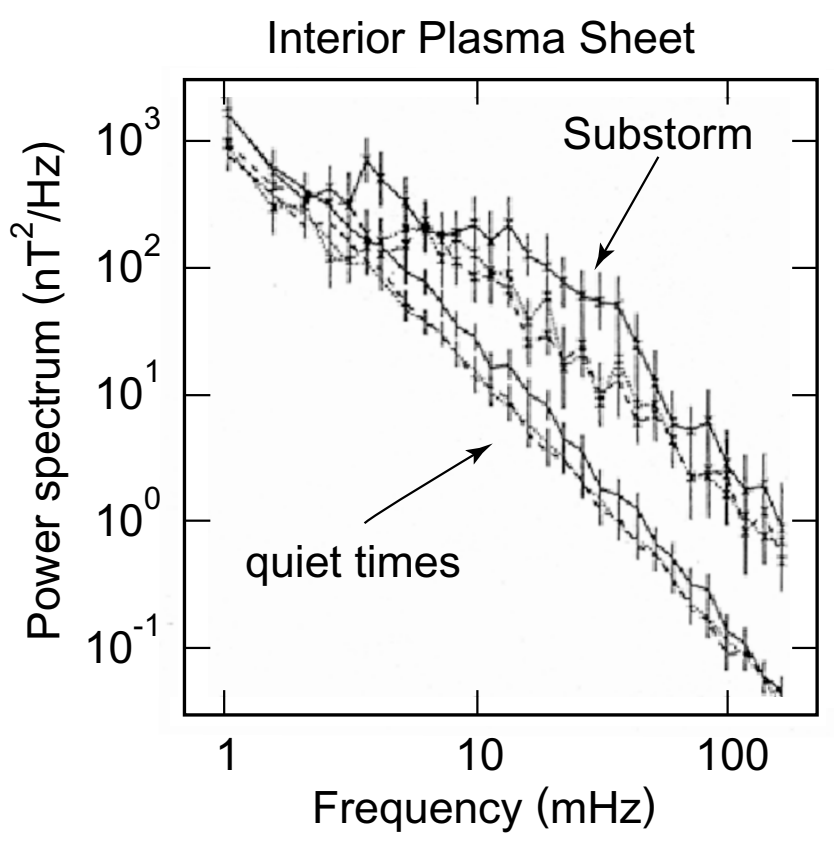

Fig. 2. Substorm and quiet time magnetic power spectra in the central plasma sheet of the Earth's magnetotail. Substorm spectra, when intense reconnection takes place in the near-Earth plasma sheet, are strongly increased at frequencies close to though below the lower-hybrid frequency. Note that it is not known whether the spacecraft has ever passed across the reconnection site or not. The spectra are in some sense averages over the central region of the plasma sheet which may or may not cover the reconnection site. The labeling is the following: dotted and dashed lines correspond to right- and left-hand polarisation, the solid line is the compressive mode. This mode clearly dominates the magnetic spectra (after Bauer et al., 1995). 


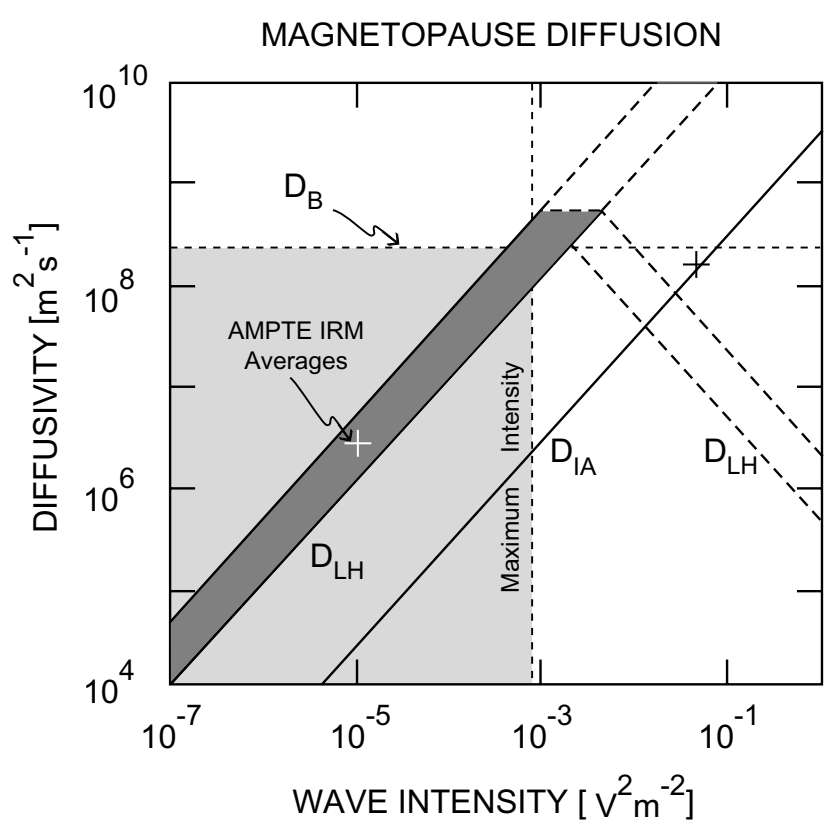

Fig. 3. Lower-hybrid drift diffusion coefficient in dependence on the electric wave intensity for reconnection at the magnetopause. The horizontal dashed line indicates the Bohm diffusion $D_{\mathrm{B}}$ limit corresponding to about $v \simeq \omega_{c e}$ as the upper limit of any reasonable anomalous collisional diffusivity for the magnetopause/low latitude boundary layer. The light cross marks the integrated electric wave field measurements at the magnetopause. The diffusivity is orders of magnitude below Bohm diffusion. For comparison, the linear ion-acoustic diffusivity $D_{\text {IA }}$ has also been drawn. The cross on this curve marks the theoretical quasi-linear estimate. It obviously overestimates the diffusivity. $D_{\text {IA }}$ for all measured intensities turns out to be weaker than $D_{\mathrm{LH}}$ which dominates in the magnetized case.

is plotted as function of $\left\langle\left|\mathbf{E}^{\mathrm{lh}}\right|^{2}\right\rangle$. The cross marks the real measurement of wave intensity while the horizontal dashed line indicates the theoretical Bohm diffusion limit which corresponds to the upper limit on any reasonable diffusion process or collision frequency $v \simeq \omega_{c e}$ equal to the electron cyclotron frequency. The width of the band around the cross corresponds to the typical average fluctuation of the intensity. The vertical dashed line terminating the shadowed region indicates the extreme upper limit of wave intensities which is occasionally measured.

The lesson learned from this figure is that the measurements never ever, except for the very rare cases of occasionally very high intensities corresponding to the vertical dashed line, support collision frequencies high enough for Bohm diffusion. The actual collision frequency is roughly two orders of magnitude lower than $\omega_{c e}$ which is not enough for global diffusive transport across the magnetopause in order to populate the low-latitude boundary layer. It is, however, high enough to account for the resistivity needed in the diffusion region when magnetopause reconnection takes place.

It can thus be argued from this result that magnetosheath plasma transport across the magnetopause is mainly if not even solely due to reconnection, and the anomalous resistivity provided by lower-hybrid drift wave turbulence is sufficient to allow for reconnection at the magnetopause. This is in agreement with the conclusions drawn from two-dimensional hybrid simulations (e.g. Winske et al., 1995) under

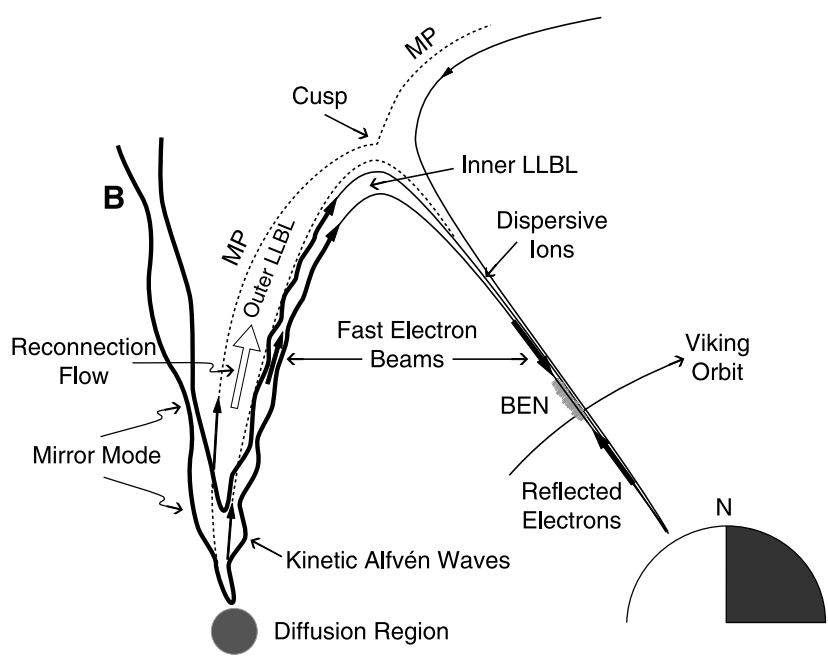

Fig. 4. Schematic representation of the site of reconnection at the dayside magnetopause. The diffusion region is located at the magnetopause. Information about its size can be inferred from measurement of electron and ion dispersion in the polar cusp at intermediate altitudes at the Viking orbit.

the conditions prevalent at the magnetopause. It is also in agreement with remote information (Fig. 4) about the size of the magnetopause diffusion region obtained from observation of electron and ion dispersion in the polar cusp region (Pottelette and Treumann, 1998).

\subsection{Cyclotron resistivity}

Since the celebrated paper of Kindel and Kennel (1971) it has been acknowledged that, in the range of current drift speeds $v_{D}$ of interest for the magnetosphere, electrostatic ion-cyclotron waves have a somewhat lower threshold for instability than ion-acoustic waves. By linear theory, the latter run unstable only for $v_{D}>c_{s}$ (see the section on ion-acoustic resistivity). This has led to the belief that ioncyclotron anomalous resistivity will dominate. The dispersion relation is $\omega^{\text {ic }} \sim \ell \omega_{c i}$ with $\omega_{c i}$ the ion-cyclotron frequency. These waves are electrostatic and propagate about perpendicular to the magnetic field. Interestingly, in the auroral magnetosphere they have been detected as quasi-harmonic waves frequently having relatively large amplitude. Dum and Dupree (1970) calculated the saturation level of the instability caused by particle orbit distortion in resonant broadening. Using their solution one readily finds that the electrostatic ion-cyclotron collision frequency is given by

$$
v_{a}^{\text {ic }} \simeq 0.56 \omega_{c i}\left(k_{\|} v_{e} / \omega_{c i}\right)
$$

which due to the smallness of $k_{\|}$is much less than $\omega_{c i}$. Hence electrostatic ion-cyclotron collisions are important in very strong magnetic fields only while not in reconnection where the magnetic field in the vicinity of the X-point is by definition small. Though ion-cyclotron waves are observed outside the reconnection site, they will do little harm to the electrons while causing some ion scattering near but outside the Xpoint and heat ions in the perpendicular direction. They are driven by the relatively strong field aligned current emanating from the already ongoing reconnection. These currents are usually carried by kinetic Alfvén waves and whistlers and play an important role in the dynamics of the plasma outside 
the X-point.

In addition, it should be repeated that ion-cyclotron resistivity is even less important because the threshold argument on ion-cyclotron and ion-acoustic waves is invalidated by nonlinear theory (Tetreault, 1991; Gray et al., 1991). Including phase space nonlinearities it turns out that below threshold before linear instability sets on ion-acoustic waves appear as ion phase space holes. These holes which are connected with large amplitude localized density fluctuations and particle heating produce anomalous resistivity at weak noncritical current drifts $v_{D}<c_{s}$. This ion-acoustic anomalous resistance is larger than the ion-cyclotron instability could ever provide. In this view ion-acoustic waves together with lowerhybrid waves are the most important in causing anomalous resistivity and rule out ion-cyclotron resistivity.

Similar arguments hold for electrostatic electron cyclotron collisions. They produce a collision frequency

$$
v_{a}^{\mathrm{ec}} \simeq 0.1 \omega_{c e}\left(v_{D} / v_{e}\right)^{3}
$$

which becomes susceptible only at extraordinarily high current drifts $v_{D} \sim v_{e}$ when the ion-acoustic or lower-hybrid instabilities are much stronger. Note also that for such speeds the Buneman instability should take over causing very strong electron heating and its side-effects.

\section{Nonlinear Evolution of Drift Waves}

As drift waves, in particular lower-hybrid drift waves, have turned out to be most important in the generation of anomalous resistivities, it is interesting to further discuss their nonlinear evolution. The above theory of lower-hybrid instability is based on quasi-linear arguments (e.g. Davidson, 1978). Drift waves are, however, known to undergo strong nonlinear evolution which in the simplest approximation follows one of the famous nonlinear equations: the Korteweg-DeVries, Kadomtsev-Petviashvili, Derivative Nonlinear Schrdinger, Musher-Sturman, Hasegawa-Mima, or Hasegawa-Wakatani equations, respectively. Either of these equations possesses a certain number of spatially localized solutions of solitary or vortex-like distortions of the plasma.

The nonlinear evolution of the lower-hybrid drift instability has been investigated by Shapiro et al. (1993) who showed that it evolves into solitons or vortices. Large numbers of such solitons or vortices correspond to a turbulent state transforming the plasma into a washing board. Electrons, when passing across the plasma, experience the distribution of the density fluctuations caused by the nonlinear drift waves and are strongly retarded. The combined action of these structures on the electron motion thus has the character of a resistive interaction. (It is interesting to note that a similar mechanism has long ago been proposed for generation of radiation in the free-electron maser, when exposing energetic electrons to a spatial density fluctuation.)

In a statistical treatment one can describe such an interaction between electrons and the turbulent plasma background via the soliton distribution function with respect to amplitude and velocity. A distribution function for soliton turbulence for drift waves was given first by Meiss and Horton (1982), based on the multiple soliton solution of the Korteweg-DeVries equation as a reasonable approximation to the Kadomtsev-Petviashvili equation. The main result of this calculation is that the spectrum of the solitary turbulence is considerably harder than that of linear drift wave turbulence. In energy space, the linear and soliton regimes exclude each other. This has been shown in Horton and Ichikawa (1996, p. 212). As a consequence, one expects that taking into account this harder spectrum in the determination of the anomalous turbulent resistivity of the plasma could lead to increased collision frequencies.

Horton and Ichikawa (1996) find for the average driftsoliton energy density

$$
\left\langle W^{\mathrm{DWS}}\right\rangle \approx 20\left(\frac{L_{T}}{L_{n}}\right)^{\frac{1}{2}}\left(\frac{r_{c s}}{L_{n}}\right)^{\frac{3}{4}}
$$

where $r_{c s}^{2}=k_{B} T_{e} / \omega_{c i}^{2}$ defines the ion-acoustic gyroradius, $L_{T}, L_{n}$ are the temperature and density gradient scales. Also, $n_{s} \approx 0.053 r_{c s}^{-1} \sqrt{L_{T} / L_{n}}\left(r_{c s} / L_{n}\right)^{1 / 4}$ is the soliton density per unit length.

The above formula shows that the soliton energy density may assume a substantial fraction of the kinetic plasma energy. In addition, measurements should exhibit spatially highly fluctuating intense drift-wave fields as is suggested by observations at the magnetopause (Fig. 3). For instance, for $L_{T} \sim L_{n}$ and the ion-sound gyroradius only $1 \%$ of the density scale one obtains for $\left\langle W^{\text {DWS }}\right\rangle \sim 0.6$ which is an enormously high value, $2-3$ orders of magnitude higher than those used in the above estimate of the lower-hybrid collision frequency. If correct, a value that high causes a local diffusion of the order of Bohm diffusion (or even stronger!). Since stronger than Bohm diffusion effectively implies free streaming, one may suspect that the simplified nonlinear theory overestimates the nonlinear effect, and the realistic resistivity will still be less than $v_{a} \leq \omega_{c e}$. On the other hand, these high collision frequencies will arise only locally. The global average solitary wave intensity will be less. Since the scale of the vortices is probably much less than the linear scale of the diffusion region, $L_{s}<\lambda_{i}, r_{c i}$, the global effect of the drift wave soliton collision frequency is smaller by the filling factor of the volume by drift wave vortices, which brings it back to realistic values of the order of those which have actually been measured.

\section{Conclusions}

The mechanisms which generate resistivity in reconnection presented in this paper suggest that of all the plenty possibilities the two remaining candidates are the ion-acoustic resistivity and the lower-hybrid drift resistivity. The former is independent of the presence of a magnetic field. It has practically no threshold when taking into account that below classical threshold $v_{D}<c_{S}$ it appears as a sequence of ion phase-space holes. In the narrow diffusion region, the typical size of which is the ion inertial length $L \sim \lambda_{i}$ or, in the magnetized case, the ion gyroradius $L \sim r_{c i}$, we believe that it can provide sufficient anomalous resistivity for reconnection. However, note that the behavior of holes is different from that of simple linear waves. Holes act locally only and in addition accelerate particles. It makes only very restricted sense to attribute simple resistivity to them. Moreover, we have given ample discussion to the problem of dependence of the ion-acoustic (and Buneman) instability on the ion-toelectron temperature ratio. This ratio makes it unlikely that 
the ion-acoustic or Buneman modes are of sufficient importance for reconnection.

The lower-hybrid drift instability (or other more general drift modes) is particularly well-suited for anomalous resistivity and transport whenever enough magnetic flux remains in the diffusion region to keep the electrons magnetized while permitting the ions to become unmagnetized. The typical scale of the diffusion region in this case is $L \sim r_{c i}$. In addition, in contrast to the ion-acoustic mode, the lower-hybrid drift instability is favored the higher the ion-to-electron temperature ratios are. This case applies realistically to the plasma sheet in the geomagnetic tail. The resistivity generated by the lower-hybrid drift instability or its nonlinear product, drift wave solitons and/or vortices, is always large enough for supporting reconnection on this scale whenever the density and temperature gradients are strong enough $L_{n} \sim r_{c i}$. It has also been found that shear motions can support the instability while very high $\beta$ will quench it. In this case a magnetic guide field remaining at the reconnection site will help to maintain the instability. Clearly such a situation applies naturally in three-dimensional reconnection thus suggesting that resistivity dominated reconnection requires a $3 \mathrm{D}$-treatment. In addition, local generation of magnetic fields in the unmagnetized reconnection diffusion region may also help. Such fields can be excited by mechanisms like the Weibel instability which makes use of intrinsic anisotropies of the distribution functions. Even in nearly complete ambient-field merging the $\mathrm{X}$-point region may then not be totally void of magnetic fields.

We may therefore conclude that, in spite of the lack of a realistic theory, anomalous resistivity does obviously not provide too big a problem for reconnection. One could argue that locally or temporally there will always be reasons for anomalous resistivity to evolve. Whether, however, such a treatment does actually meet the real physics of reconnection, is a question that is not touched by asking for mechanisms that can generate enough anomalous resistivity. When this happens, reconnection will set on locally and temporally. Localization then implies that the expected type of reconnection will fall into the family of some variant of Petschek reconnection.

The general fluid-Ohm's law also provides other possibilities which violate the frozen-in concept. One particularly important case is whistler mode turbulence implicit to the Hall current terms. It causes pitch-angle diffusion which, in the fluid description, produces anomalous viscosity. This leads to non-resistive spatial diffusion of particles across the magnetic field. Viscosity can formally be translated into collision frequencies as well. However, the mechanism is different from direct ordinary or anomalous resistivity. In pitchangle scattering particle energy remains about unchanged. There is apparent friction in elastic collisions between particles and waves, with viscosity arising due to variation of the gyroradius of the particles and finite-gyro radius effects. It is basically this effect which helps decoupling the particles from the field and therefore contributes to reconnection whenever electron whistlers are excited. Clearly, this is a very important alternative to ordinary resistive reconnection.

A remaining question is of how whistler turbulence will be excited in reconnection. In the region where the plasma is still magnetized this can be accomplished by either anisotropic electron distributions with $T_{e \perp}>T_{e \|}$, by field-aligned electron beams, and by density gradients. In the latter case the whistler propagates near the resonance cone on the magnetized branch of the lower-hybrid mode, in which case it causes anomalous resistance similar to the lower-hybrid mechanism. Whistler mode noise generated by electron beams (e.g. auroral hiss, see Maggs, 1976, 1989) is weak and broadband and is not expected to be of importance. Moreover, it supposes the presence of fast electron beams generated in the reconnection process. On the other hand, anisotropy-generated whistlers require the maintenance of temperature anisotropy. This is not unreasonable, but the effect will be that the pitch-angle scattering of the resonant electrons proceeds in the direction parallel to the magnetic field, decreasing their gyro-radii. It thus seems that only the first mechanism contributes to anomalous resistance. As argued by Drake (1995) and Shay et al. (1999), the most important action of whistler turbulence will thus probably not be anomalous resistance but the evolution of nonlinear structure in the whistler field on scales of the electron inertial length $\delta_{e}=c / \omega_{p e}$.

Another alternative is localized collisionless annihilation of the magnetic field. This can be provided by the diamagnetic effect of particles trapped in the environment of the Xline, an effect which is caused by mirror mode instability. In such a case the frozen-in condition is set out of action in a way different from resistive or viscous diffusion. Reconnection then turns out to be a phase transition in a high-temperature plasma near a thermodynamic equilibrium state. This mechanism has been proposed recently (Treumann and Sckopke, 1999) but needs to be explored in detail.

Thus, whether or not resistivity is really necessary for reconnection in plasma cannot be definitively decided even at the present time, nearly half a century after the invention of reconnection. The personal opinion of this author is that there are several different types of reconnection: resistive reconnection where resistivity is given a priori, reconnection driven by anomalous resistivity, and genuinely collisionless reconnection caused by anomalous Hall viscosity or other kinetic effects. The latter require collisionless full particle simulation treatments. An ultimate answer may even require three-dimensional full particle simulations. Possibly of these three types only the anomalously resistive and the genuinely collisionless mechanisms apply to the cosmic plasma in near Earth space.

We have dealt here with resistive reconnection while not mentioning another important case suggested by the generalized Ohm's law Eq. (1), viz. turbulent mixing contained in the second term on the rhs. Even in the incompressible case a term $\mathbf{v} \cdot \nabla \mathbf{j}$ is refained in Ohm's law. Numerical simulations of reconnection have shown that the current and velocity fields become highly turbulent on the electron as well as on the ion scales and larger, while the magnetic field remains fairly regular. Such turbulence will stir the plasma on meso-scales. In the presence of even a very weak microscopic diffusion acting on very small scales it is then sufficient for breaking the frozen-in condition when the plasma during the stirring (eddy) period displaces from one flux tube to a neighbouring one. This implies meso-scale transport caused by micro- 
diffusion and decouples the plasma from the field.

Acknowledgments. I thank the organizers of MR2000 for the invitation. M. Hoshino, M. Ono, G. Paschmann, T. Phan, M. Scholer, B. Sonnerup and T. Terasawa are thanked for illuminating discussions. The contributions of K. Arzner, T. M. Bauer, J. LaBelle and R. Pottelette are gratefully acknowledged. This work has been performed during a visitors period at ISSI, Bern. I thank its directors J. Geiss, G. Paschmann, and R. V. Steiger for their interest and support and the ISSI staff for its hospitality. Part of this work falls under a PROCOPE program under contract number D/9822921. I dedicate this paper to my late colleague Norbert Sckopke, who untimely passed away midst in our discussions on November 28, 1999.

\section{References}

Arzner, K. and M. Scholer, Magnetotail reconnection: simulation predictions on magnetic time series, Earth Planets Space, 53, this issue, 655661,2001

Bauer, T. M., W. Baumjohann, R. A. Treumann, and N. Sckopke, Lowfrequency waves in the near-Earth plasma sheet, J. Geophys. Res., 100, 9605-9617, 1995.

Baumjohann, W., R. A. Treumann, J. LaBelle, and R. R. Anderson, Average electric wave spectra across the plasma sheet and their relation to ion bulk speed, J. Geophys. Res., 94, 15221-15230, 1989.

Baumjohann, W., R. A. Treumann, and J. LaBelle, Average electric wave spectra across the plasma sheet: Dependence on ion density and ion beta, J. Geophys. Res., 95, 3811-3817, 1990.

Brizard, A. J., Nonlinear gyrokinetic Maxwell-Vlasov equations using magnetic co-ordinates, J. Plasma Phys., 41, 541-559, 1989.

Brizard, A. J., Variational principle for nonlinear gyrokinetic VlasovMaxwell equations, Phys. Plasmas, 7, 4816-4822, 2000.

Cai, H. J., D. Q. Ding, and L. C. Lee, Momentum transport near a magnetic X line in collisionless reconnection, J. Geophys. Res., 99, 35-42, 1994.

Chen, L., Theory of plasma transport induced by low-frequency hydromagnetic waves, J. Geophys. Res., 104, 2421-2428, 1999.

Cheng, C. Z., A kinetic-magnetohydrodynamic model for low-frequency phenomena, J. Geophys. Res., 96, 21259, 1991.

Cheng, C. Z. and J. R. Johnson, A kinetic-fluid model, J. Geophys. Res., 104, 413, 1999.

Davidson, R. C., Quasi-linear stabilization of lower-hybrid drift instability, Phys. Fluids, 21, 1375-1380, 1978.

Drake, J. F., Magnetic reconnection: A kinetic treatment, in Physics of the Magnetopause, edited by P. Song, B. U. Ö. Sonnerup, M. F. Thomsen, Geophys. Monogr. 90, p. 155, American Geophys. Union, Washington, D.C., 1995.

Drake, J. F., R. G. Kleva, and M. E. Mandt, The structure of thin current layers: Implications for magnetic reconnection, Phys. Rev. Lett., 73, 1251-1254, 1994.

Dum, C. T., Anomalous heating by ion-sound turbulence, Phys. Fluids, 21, 945-969, 1978.

Dum, C. T. and T. C. Dupree, Nonlinear stabilization of high-frequency instabilities in a magnetic field, Phys. Fluids, 13, 2064-2081, 1970.

Frieman, E. A. and L. Chen, Nonlinear gyrokinetic equations for lowfrequency electromagnetic waves in general plasma equilibria, Phys. Fluids, 25, 502-508, 1982.

Gray, P., M. K. Hudson, W. Lotko, and R. Bergmann, Decay of ion beam driven acoustic waves into ion holes, Geophys. Res. Lett., 18, 1675-1678, 1991.

Hollweg, J. and H. Völk, New plasma instabilities in the solar wind, $J$.
Geophys. Res., 75, 5297-5312, 1970.

Horton, W. and Y.-H. Ichikawa, Chaos and Structures in Nonlinear Plasmas, 340 pp., World-Scientific, Singapore, 1996.

Hoshino, M., A. Nishida, T. Yamamoto, and S. Kokubun, Turbulent magnetic field in the distant magnetotail: Bottom-up process of plasmoid formation?, Geophys. Res. Lett., 21, 2935-2938, 1994.

Johnson, J. R. and C. Z. Cheng, Global structure of mirror modes in the magnetosheath, J. Geophys. Res., 102, 7179-7189, 1997.

Kindel, J. F. and C. F. Kennel, Topside current instabilities, J. Geophys. Res., 76, 3055-3071, 1971.

Krall, N. A. and P. C. Liewer, Low-frequency instabilities in magnetic pulses, Phys. Rev. A, 4, 2094-3003, 1971.

Krall, N. A. and A. W. Trivelpiece, Principles of Plasma Physics, 673 pp., McGraw-Hill, New York, 1973.

Liewer, P. C. and N. A. Krall, Self-consistent approach to anomalous resistivity applied to theta pinch experiments, Phys. Fluids, 16, 1953-1963, 1973.

Lee, N. C. and G. K. Parks, Ponderomotive force in a nonisothermal plasma, Phys. Fluids, 31, 90-95, 1988.

Lund, E. J., R. A. Treumann, and J. LaBelle, Quasi-thermal fluctuations in a beam-plasma system, Phys. Plasmas, 3, 1234-1240, 1996.

Maggs, J. E., Coherent generation of VLF hiss, J. Geophys. Res., 81, 17071721,1976

Maggs, J. E., Nonlinear evolution of the auroral electron beam, J. Geophys. Res., 94, 3631-3640, 1989.

Meiss, D. A. and W. Horton, Jr., Drift-wave turbulence from a soliton gas, Phys. Rev. Lett., 48, 1362-1365, 1982.

Newman, D. L., M. V. Goldman, and R. E. Ergun, Langmuir turbulence in moderately magnetized space plasmas, Phys. Plasmas, 1, 1691-1699, 1994.

Pottelette, R. and R. A. Treumann, Impulsive broadband electrostatic noise in the cleft: A signature of dayside reconnection, J. Geophys. Res., 103, 9299-9307, 1998.

Robinson, P. A., Nonlinear wave collapse and strong turbulence, Rev. Mod. Phys., 69, 507-573, 1997.

Sagdeev, R. Z., Cooperative phenomena and shock waves in collisionless plasmas, Rev. Plasma Phys., 4, 23-91, 1966.

Sagdeev, R. Z., The Oppenheimer Lecture: Critical problems in plasma physics, Rev. Mod. Phys., 51, 1-20, 1979.

Shapiro, V. D., V. I. Shevchenko, G. I. Solov'ev, V. P. Kalinin, R. Bingham, R. Z. Sagdeev, M. Ashour-Abdalla, J. Dawson, and J. J. Su, Nonlinear evolution and collapse of lower-hybrid drift waves, Phys. Fluids B, 5, 3148-3162, 1993.

Shay, M. A., J. F. Drake, B. N. Rogers, and R. E. Denton, The scaling of collisionless, magnetic reconnection for large systems, Geophys. Res. Lett., 26, 2163-2166, 1999.

Tetreault, D., Theory of electric fields in the auroral acceleration region, $J$. Geophys. Res., 96, 3549-3561, 1991.

Treumann, R. A. and W. Baumjohann, Advanced Space Plasma Physics, pp. 381, Imperial College Press, London, 1997.

Treumann, R. A. and N. Sckopke, The collisionless mirror mode in the magnetosheath: A superconducting analogue, Eos Trans. AGU, 80, Fall Meet. Suppl., SM-237, 1999.

Winske, D., V. Thomas, and S. P. Gary, Diffusion at the magnetopause: The theoretical perspective, in Physics of the Magnetopause, edited by $\mathrm{P}$. Song, B. U. Ö. Sonnerup, M. F. Thomsen, Geophys. Monogr. 90, p. 337, American Geophys. Union, Washington, D.C., 1995.

R. A. Treumann (e-mail: tre@mpe.mpg.de) 